\title{
Cancer screening: from uptake to informed decision making
}

\author{
Fiona Godlee editor in chief
}

The BMJ

Colorectal cancer is the third most common cancer worldwide and the second most common cause of cancer related death. In developed countries one in 20 people will develop the disease. Given this major burden, establishing screening programmes has been a public health priority. People at average risk are generally offered screening once they reach 50 , but there is no consensus on which tests to use or how often. The Rapid Recommendations team has now taken up this issue, asking whether screening makes an important difference to health outcomes, and which screening test is best (doi:10.1136/bmj. 15515).

Their methods will be familiar to regular readers but are worth reiterating, because they offer a fresh approach to providing trustworthy and timely guidance. Triggered by new evidence that is potentially practice changing (in this case, updates from three major randomised trials), they convene a panel of experts that includes patients as full members (in this case, three people who have experienced screening for colorectal cancer). All panel members are required to be free of relevant financial conflicts of interest. They systematically review the evidence and develop guidance, taking a range of transparently described approaches to deal with gaps in the evidence and to incorporate patients' values and preferences.

This panel looked at four different screening schedules: annual and biennial faecal immunochemistry testing (FIT), single sigmoidoscopy, and single colonoscopy, each compared with no screening. They were confronted with an interesting challenge: while there have been randomised trials of sigmoidoscopy, there are none yet of colonoscopy or FIT. So they combined standard systematic review and meta-analysis (doi:10.1136/bmjopen-2019-032773) with microsimulation modelling (doi:10.1136/bmj.15383). This involves estimates based on simulated life histories from several large population cohorts. They also looked at the harms and burden of screening, and explored the level of benefit people would expect before agreeing to be screened.

They find that screening does not reduce all cause mortality whichever test is used, but it does reduce cancer specific mortality. Acknowledging the uncertainties arising from the lack of strong evidence, they recommend that screening be offered only to people with a risk of colorectal cancer of $3 \%$ or more, as assessed by tools such as the QCancer calculator (https: //qcancer.org), and that the choice of test be left to the individual's personal preference.

This personalised and risk based approach may seem obvious. But it represents a radical shift, says Philippe Autier in his linked editorial (doi:10.1136/bmj.15558). Screening programmes measure their success by the number of people who take part. What is being recommended here will very likely reduce uptake. In future, screening programmes should be judged not on uptake but on the quality of informed decision making. 